\title{
Was leistet die funktionelle Bildgebung für die Evaluation und Weiterentwicklung der Verhaltenstherapie?
}

\author{
Herta Flor
}

Institut für Neuropsychologie und Klinische Psychologie, Zentralinstitut für Seelische Gesundheit, Universität Heidelberg, Mannheim, Deutschland

\section{Schlüsselwörter \\ Psychotherapie - Funktionelle Bildgebung · Gehirn · Schmerz · Abhängigkeit}

\section{Einleitung}

Die funktionelle Bildgebung des Gehirns, zu der ich neben der Magnetresonanztomographie (MRT) und der Positronenemissionstomographie auch die Multikanal-Elektroenzephalographie (EEG) und die Magnetenzephalographie (MEG) zählen möchte, hat in den letzten Jahren auch in der klinischen Psychologie an Akzeptanz gewonnen. Diese Methoden eignen sich derzeit sicher nicht zur Individualdiagnostik - dafür ist die interindividuelle Varianz zu groß. Sie geben jedoch Hinweise auf pathogenetische Aspekte, die bislang mit Verhaltensbeobachtungen und Methoden, die auf subjektiven Einschätzungen basieren, nicht erfasst werden können. Außerdem können sie in der Evaluation von Therapieverfahren sinnvoll sein. Ich möchte im Folgenden anhand von Beispielen aus unserer eigenen Forschung zeigen, welche neuen Erkenntnisse aus solchen Verfahren für verhaltenstherapeutische Interventionen gewonnen werden können.

\section{Weiterentwicklung therapeutischer Interventionen}

Untersucht man bei abstinenten Abhängigen das Drogenverlangen oder die Bewertung von mit der Droge assoziierten Reizen, so kann man aus diesen Maßen nicht vorhersagen, wer später rückfällig werden wird. Jedoch hat der Einsatz des EEGs bzw. der funktionellen MRT (fMRT) gezeigt, dass bei abhängigen Personen die positive Verarbeitung von suchtrelevanten Reizen ein entscheidendes Charakteristikum der Abhängigkeit ist - wichtiger als die negativen Aspekte des

\section{Key Words}

Behavioral treatment - Functional imaging - Brain · Pain . Addiction

Entzugs. So können das Drogenverlangen als niedrig und drogenrelevante Reize nach dem Entzug als aversiv eingeschätzt werden, aber physiologische Maße dennoch eine positive Evaluation der Reize nahelegen [Grüsser et al., 2002; Wölfling et al., 2008]. Je positiver die physiologische Antwort auf die Reize war, z.B. je ausgeprägter die langsamen Potentiale des EEG auf die drogenrelevanten Reize und je intensiver die Aktivierung des ventralen Striatums in einer fMRT-Untersuchung, desto wahrscheinlicher war ein späterer Rückfall bei abstinenten Alkoholikern [z.B. Grüsser et al., 2004]. Diese Befunde haben unmittelbare Relevanz für die Therapie, da es nach diesen Daten notwendig ist, die positiven Aspekte der Drogeneinnahme zu entwerten und nicht auf negative Effekte zu fokussieren. Ein entsprechend verändertes Verhaltenstraining war insbesondere bei schwer abhängigen Patienten erfolgreicher als die für gewöhnlich eingesetzte kognitive Verhaltenstherapie [Löber et al., 2006].

\section{Entwicklung innovativer Therapien}

Für chronische Erkrankungen, die mit Lern- und Gedächtnisprozessen assoziiert sind und mit plastischen Veränderungen des Gehirns einhergehen, wie z.B. chronische Schmerzen, Tinnitus oder Dystonie, wurden völlig neue Interventionen entwickelt, die zum Ziel haben, die unangepassten Lern- und Gedächtnisprozesse und die maladaptive Gehirnplastizität zu verändern. So konnte man zeigen, dass sensorisches Diskriminationstraining oder Spiegeltherapie effektive Therapien für Phantomschmerz sind [vgl. Diers, 2009]. Beim Phantom-

\section{KARGER}

Fax +497614520714

Information@Karger.de

www.karger.com (c) 2009 S. Karger GmbH, Freiburg

Accessible online at:

www.karger.com/ver 
schmerz ließ sich mittels bildgebender Verfahren nachweisen, dass er eng mit der Verschiebung der Repräsentation des von der Amputation betroffenen Körperteils im somatosensorischen und motorischen Kortex assoziiert ist. Beim sensorischen Diskriminationstraining müssen Patienten Reize am Stumpf diskriminieren lernen. Durch dieses verhaltensrelevante Training bildet sich die Einwanderung von Nervenimpulsen in das Repräsentationsareal des nicht mehr vorhandenen Gliedes zurück. Erfolge beim Training, Veränderungen im Phantomschmerz und in der Repräsentation im Gehirn sind hier hoch korreliert und können von Placeboeffekten abgegrenzt werden [Flor et al., 2001]. Beim Spiegeltraining wird das intakte Glied vor einem Spiegel so bewegt, dass man im Spiegel die Bewegung des nicht mehr vorhandenen Körperteils zu sehen glaubt. Dieses Training kann den Phantomschmerz sehr effektiv vermindern und reaktiviert die Gehirnregion, in die vorher Nervenzuflüsse aus dem amputierten Glied flossen. Weiterentwicklungen dieser Therapien sind Gehirn-Computer-Interfaces und Rückmeldeanordnungen mit virtueller Realität, die Hirnprozesse mit negativen Empfindungen noch intensiver beeinflussen können.

\section{Evaluation von Psychotherapiewirkungen und -mechanismen}

Ein weiterer positiver Aspekt bildgebender Verfahren in der Psychotherapie ist die Möglichkeit, die Effektivität von Therapien zu überprüfen und genaueren Aufschluss über Wirkmechanismen von Therapien zu erhalten. Insbeson- dere zu Phobien, Zwangsstörungen und Depression liegen bereits eine Reihe wegweisender Arbeiten vor [vgl. Linden, 2008], die zeigen konnten, dass Psychotherapien Hirnveränderungen, die mit diesen Störungen assoziiert sind, rückgängig machen können. Darüber hinaus konnten Vergleiche der Hirnveränderungen nach Gabe von Antidepressiva oder nach Psychotherapie differenzielle Effekte dieser Verfahren, aber auch eine Reihe gleicher Veränderungen nachweisen und Hinweise für Wirkmechanismen geben. Bei Patienten mit Fibromyalgie fanden wir, dass ein verhaltenstherapeutisches Extinktionstraining die vorher defizitäre Aktivierung der Insula, einer wichtigen schmerzmodulierenden Hirnstruktur, erhöhen konnte und dass das Ausmaß der Inselaktivierung hoch mit der Abnahme der Beeinträchtigung durch den Schmerz korrelierte [Diers et al., unveröffentlichte Daten].

\section{Wirkungen auf die Akzeptanz von Psychotherapie}

Schließlich können die Befunde der Bildgebung, die funktionelle und strukturelle Hirnveränderungen durch Psychotherapie nachweisen, auch die Akzeptanz von Psychotherapie im Vergleich zu Pharmakotherapie erhöhen. Insbesondere vergleichende Studien mit Pharmakotherapie könnten zusätzliche Belege für die Gleichwertigkeit bzw. häufig die Überlegenheit von Psychotherapie erbringen, insbesondere wenn gezeigt werden kann, dass sich Biomarker der Störung beeinflussen lassen.

\section{Literatur}

Diers M: Funktionelle Bildgebung bei chronischen

Schmerzerkrankungen: Implikationen für die Therapie. Verhaltenstherapie 2009;19:86-93.

Flor H, Denke C, Schäfer M, Grüsser S: Effects of

sensory discrimination training on cortical reorganization and phantom limb pain. Lancet 2001;357: $1763-1764$.

Grüsser SM, Heinz A, Raabe A, Wessa M, Podschus

J, Flor H: Stimulus-induced craving and startle potentiation in abstinent alcoholics and controls. Eur Psychiatry 2002;17:188-193.
Grüsser SM, Wrase J, Klein S, Hermann D, Smolka MN, Ruf M, Weber-Fahr W, Flor H, Mann K, Braus DF, Heinz A: Cue-induced activation of the striatum and medial prefrontal cortex is associated with subsequent relapse in abstinent alcoholics. Psychopharmacology (Berl) 2004;175:296-302.

Linden DE: Brain imaging and psychotherapy: me-

thodological considerations and practical implications. Eur Arch Psychiatry Clin Neurosci 2008;258 (suppl 5):71-75.
Loeber S, Croissant B, Heinz A, Mann K, Flor H: Cue - exposure in the treatment of alcohol dependence: effects on drinking outcome, craving, and self-efficacy. Br J Clin Psychol 2006;45:515-529.

Wölfling K, Flor H, Grüsser SM: Psychophysiologi-

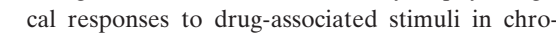
nic heavy cannabis use. Eur J Neurosci 2008;27: 976-983. 